Methods Simulation of electronic prescription (10 physicians), preparation/administration (10 paediatric/8 adult intensive care nurses) of 5 drugs (midazolam, fentanyl, noradrenaline, ketamine, furosemide) for 15 fictive patients (different dosage/ weight). Two-sessions study (VarC vs StdC, each 150 prescriptions/270 preparations). Issues: time (mean $\pm \mathrm{SD}$ in seconds); precision (target deviation in\%, mediane [IQR]) of drug concentration (quantitative analysis), dose and rate (calculated by nurses).

Results With StdC, prescription time was significantly longer $(72 \pm 36$ vs $86 \pm 32, \mathrm{p}<0.001)$ and preparation/administration time shorter $(286 \pm 98$ vs $216 \pm 93, \mathrm{p}<0.0001)$. Precision of drug concentration was increased (4.4\% [2.0 to 11.5 ] vs $4.1 \%$ [1.6 to 8.4], $\mathrm{p}=0.004)$ with a reduction of concentrations $>20 \%(44 / 270(16.3 \%)$ vs $23 / 270(8.5 \%), p=0.005)$. Precision of dose was decreased $4.4 \%$ [2.0 to 11.1 ] vs $11.8 \%$ [5.1 to $23.3], \mathrm{p}<0.0001)$ with an increase of dose $>20 \%(42 / 270$ $(15.6 \%)$ vs $83 / 270(30.7 \%), \mathrm{p}<0.0001)$. Precision of rate was decreased $(0.0 \%$ [ 0 to 0 ] vs $6.8 \%$ [3.2 to 20.6], $\mathrm{p}<0.0001)$. No association with age, years of experience, number of worked hours before study, paediatric or adult ICU nurses was observed on precision.

Conclusions Preparation time and drug concentration precision was strongly improved with StdC. Strategies to deal with prescription time and poor dose and rate precision should be considered before moving to StdC.

\section{PS-133 THE CARDIAC OUTPUT (CO) MONITORING IN CHILDREN WITH ACUTE CIRCULATORY FAILURE (ACF) IN PAEDIATRIC INTENSIVE CARE UNIT (PICU): OESOPHAGEAL DOPPLER (OD) VERSUS TRANSTHORACIC ECHOCARDIOGRAPHY (TTE)}

K Elhalimi, MA Negadi, H Bouguetof, D Boumendil, Z Mentouri. Faculty of Medicine of Oran, CHU Oran, Oran, Algeria

\subsection{6/archdischild-2014-307384.427}

Background and aims $\mathrm{CO}$ monitoring has an important role for management of ACF in PICU. This monitoring device tracks the changes in $\mathrm{CO}$ induced by volume expansion or inotropic drugs. In this way $\mathrm{CO}$ can be measured noninvasively using aortic blood flow (ABF), continuously at the descending thoracic aorta with OD or discontinued in ascending thoracic aorta with TTE.

The aim of this study is to compare the CO obtained by TTE and OD in the management of ACF in PICU.

Methods A prospective and comparative study conducted in PICU between march 2012 and march 2014.

We investigate 16 mechanically ventilated children less than 1 year who had tachycardia, hypotension, oliguria, delayed capillary refilling or haemodynamic instability despite vasopressor drugs, we compare the measurements of the $\mathrm{CO}$ and strong volume (SV) obtained by OD 'ATYS-WAKI 2' and TTE 'SCHIMADZU SDU 2200 PRO' before and after volume expansion (VE).

Results 32 paired (CO and SV) measurements were obtained: a strong correlation was found between CO obtained by OD and by TTE before and after VE (Index of Pearson: $\mathrm{R}^{2}=0,983, \mathrm{R}^{2}$ $=0.977)$. The same correlation between the SV obtained by OD and by TTE was observed before and after VE respectively (Index of Pearson: $\mathrm{R}^{2}=0.982, \mathrm{R}^{2}=0.983$ ).

Conclusion $\mathrm{OD}$ is an appropriated, very simple and noninvasive method to measure CO. This technique remains reliable and reproducible comparative to TTE to guide VE.

\section{PS-134 PRISM SCORE AND NONINVASIVE VENTILATION (NIV) FOR ACUTE RESPIRATORY FAILURE}

I Bakalli, E Celaj, E Kola, R Lluka, S Sallabanda. PICU, UHC Mother Teresa, Tirana, Albania

\subsection{6/archdischild-2014-307384.428}

Introduction PRISM score (Paediatric risk mortality) is widely used to determine the risk of mortality in children in PICU. Recent studies had found correlation between the low values of PRISM with the success of noninvasive ventilation (NIV) and the high values with failure, but without clearly defined who will be called higher or lower value of PRISM score.

Objective To evaluate the predictive value of PRISM score for NIV success in acute respiratory failure (ARF).

Methods This is a prospective study. Are included all children admitted at PICU during January-December 2011. NIV was used as the primary support for ARF. We analysed the predictive value of the PRISM score using ROC curves and the trend of success change by Chi-square trend.

Results A total of 42 patients were included. NIV success rate was $73.8 \%$. Prism score in the success group was $9.5 \pm 3.9$ vs. $14.5 \pm 6.6$ points in the failure group $(\mathrm{p}=0.0184)$. Max value was 27 points, min value 3 points. By ROC curves, PRISM $<10$ points before NIV results significant predictive factor for NIV success with predictive positive value $87.5 \%$. By Chi-square trend it was found a significant trend of success reduction with increasing value of PRISM. For PRISM score $=10$ up to 15 points, OR $=0.3(95 \%$ CI $0.05-2.0) \mathrm{p}=0.2$. For PRISM score $>15$ points, $\mathrm{OR}=0.08(95 \%$ CI $0.01-0.5) \mathrm{p}=0.01 .\left(\chi_{\text {for linear }}^{2}\right.$ trend $=7.6, \mathrm{p}<0.01)$.

Conclusion PRISM score $<10$ points is a significant predictive factor for NIV success. For PRISM score $>15$ points the likelihood to have success is decreased significantly.

\section{PS-135 CONSENSUS DEFINITIONS, LIMITING VALUES AND RECOMMENDATIONS ON INTRA-ABDOMINAL HYPERTENSION THERAPY (IAH) AND ABDOMINAL COMPARTMENT SYNDROME (ACS) IN CHILDHOOD PUBLISHED BY THE WSACS-PAEDIATRIC GUIDELINES-COMMITTEE}

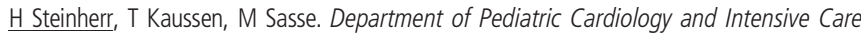
Medicine, Hannover Medical School, Hannover, Germany

\subsection{6/archdischild-2014-307384.429}

Background and aim Hitherto, neither evidence-based definitions nor age-related recommendations existed on the diagnosis and treatment of Intra-Abdominal Hypertension (IAH) and Abdominal Compartment Syndrome (ACS) in childhood. Following their 7th World Congress 2011 in Orlando (Florida), the World Society of the Abdominal compartment ayndrome (www. WSACS.org) instructed a paediatric expert committee to develop appropriate guidelines.

Methods Based on a systematic database search relevant literature was identified related to neonatal and paediatric IAH/ACS. Using a modified Delphi methodology according to the GRADE model (A to D), all papers were checked with respect to their validity and evidence. Afterwards, paediatric consensus definitions and recommendations were framed.

Results Results were published in intensive care medicine together with the revised 2013 consensus guidelines for adults (ICM 2013; 39(7):1190-206). Besides general definitions, risk factors and critical IAP thresholds (IAP: intra-abdominal 